\title{
Processing of Sliding Spotlight and TOPS SAR Data Using Baseband Azimuth Scaling
}

\author{
Pau Prats, Member, IEEE, Rolf Scheiber, Josef Mittermayer, Member, IEEE, \\ Adriano Meta, Member, IEEE, and Alberto Moreira, Fellow, IEEE
}

\begin{abstract}
This paper presents an efficient phase preserving processor for the focusing of data acquired in sliding spotlight and Terrain Observation by Progressive Scans (TOPS) imaging modes. They share in common a linear variation of the Doppler centroid along the azimuth dimension, which is due to a steering of the antenna (either mechanically or electronically) throughout the data take. Existing approaches for the azimuth processing can become inefficient due to the additional processing to overcome the folding in the focused domain. In this paper, a new azimuth scaling approach is presented to perform the azimuth processing, whose kernel is exactly the same for sliding spotlight and TOPS modes. The possibility to use the proposed approach to process data acquired in the ScanSAR mode, as well as a discussion concerning staring spotlight, is also included. Simulations with point targets and real data acquired by TerraSAR-X in sliding spotlight and TOPS modes are used to validate the developed algorithm.
\end{abstract}

Index Terms-Azimuth scaling, SAR processing, ScanSAR, spotlight, synthetic aperture radar (SAR), Terrain Observation by Progressive Scans (TOPS).

\section{INTRODUCTION}

$\mathbf{S}$ PACEBORNE synthetic aperture radar (SAR) systems have usually had the capability to operate in stripmap and ScanSAR imaging modes. Later on, the use of antennas electronically steered also in the azimuth dimension allowed an efficient implementation of the spotlight mode and of the recently developed wide-swath Terrain Observation by Progressive Scans (TOPS) [1] mode. A clear example of this versatility is the TerraSAR-X satellite [2], which can acquire images in stripmap, spotlight, and ScanSAR modes and has also acquired images in the TOPS mode [3].

New imaging modes demand efficient processing algorithms. Typically, stripmap processors have been adapted to the needs of the different modes by adding pre- and/or postprocessing steps [1], [4], [5], or new approaches have been developed [6][10]. This paper proposes a unified approach for the processing of sliding spotlight and TOPS imaging modes. In particular, the new contribution focuses on the processing of the azimuth sig-

Manuscript received February 9, 2009; revised May 27, 2009. First published September 29, 2009; current version published January 20, 2010. This work was supported in part by the European Space Agency under Contract C20679/07/NL/CB.

P. Prats, R. Scheiber, J. Mittermayer, and A. Moreira are with the Microwaves and Radar Institute, German Aerospace Center (DLR), 82234 Wessling, Germany (e-mail: Pau.Prats@dlr.de; rolf.scheiber@dlr.de; josef.mittermayer@ dlr.de; alberto.moreira@ dlr.de).

A. Meta was with the Microwaves and Radar Institute, German Aerospace Center (DLR), 82234 Munich, Germany. He is now with MetaSensing, 2201 Noordwijk, The Netherlands (e-mail: adriano.meta@metasensing.com).

Digital Object Identifier 10.1109/TGRS.2009.2027701 nal, assuming that range cell migration (RCM) has already been corrected. To this purpose, a new azimuth scaling approach, called baseband azimuth scaling (BAS), has been developed.

The sliding spotlight and TOPS modes perform a steering of the antenna (either mechanically or electronically) throughout the data take. Consequently, the total azimuth signal bandwidth might span over several pulse repetition frequency (PRF) intervals. An efficient solution to overcome this insufficient sampling is the use of subapertures [7]. This approach takes advantage of the higher PRF with respect to the instantaneous azimuth bandwidth, allowing the selection of (in many cases) large portions of the raw data to perform the range-variant processing. Afterward, the subapertures are recombined so that only the full resolution azimuth focusing is left. However, if classical matched filtering is used, a large reference function is needed, provided that the azimuth spectrum has been extended to accommodate for the whole azimuth bandwidth. This turns out to be an inefficient solution. Azimuth scaling with spectral analysis (SPECAN) was proposed in [7] in order to avoid the use of a large reference function. However, some inconveniences arise in the sliding spotlight mode. First, extra steps are needed in order to accurately perform the azimuth weighting, and, second, folding in the focused domain (i.e., wrap around in the azimuth dimension) might result when using the SPECAN approach. Similarly, the TOPS mode will result, in most cases, in folding in time when using SPECAN.

This paper is organized as follows. Section II reviews the two imaging modes, analyzing the properties of the azimuth signal, whereas Section III presents the analytical derivation of BAS. Section III-C addresses the possibility to process the ScanSAR data using the proposed algorithm. In this case, the approach becomes less efficient than the existing algorithms, e.g., [6], but still more efficient than the direct solution consisting of a large time extension before azimuth compression. A comment is also given concerning staring spotlight in Section III-D, where it is shown that the proposed approach turns unsuitable with such a mode. Finally, Section IV presents the results using simulated point targets and real data acquired by TerraSAR-X in sliding spotlight and TOPS modes.

\section{IMAGING MODES}

\section{A. Sliding Spotlight}

The staring spotlight mode consists in the steering of the antenna so that the main beam is always pointing at the scene center. Doing so, the illumination time is increased, and, 


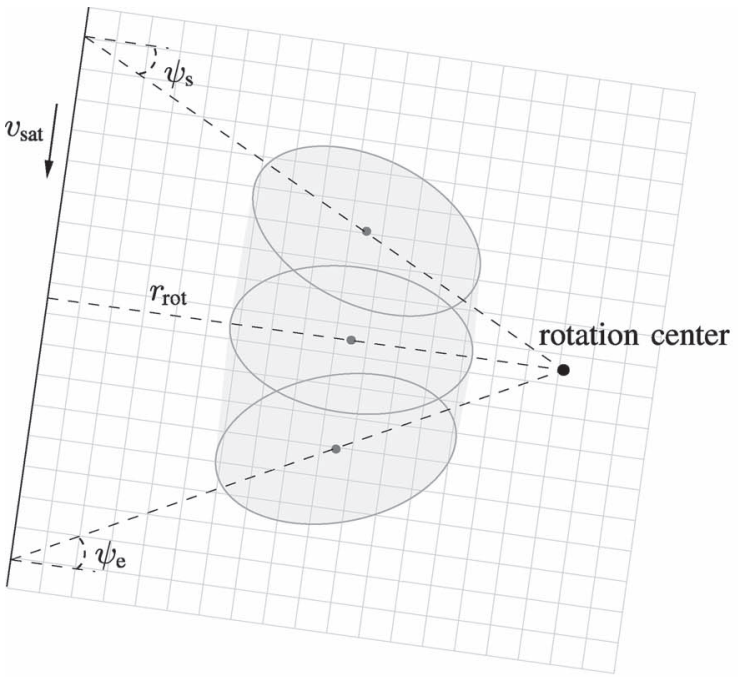

(a)

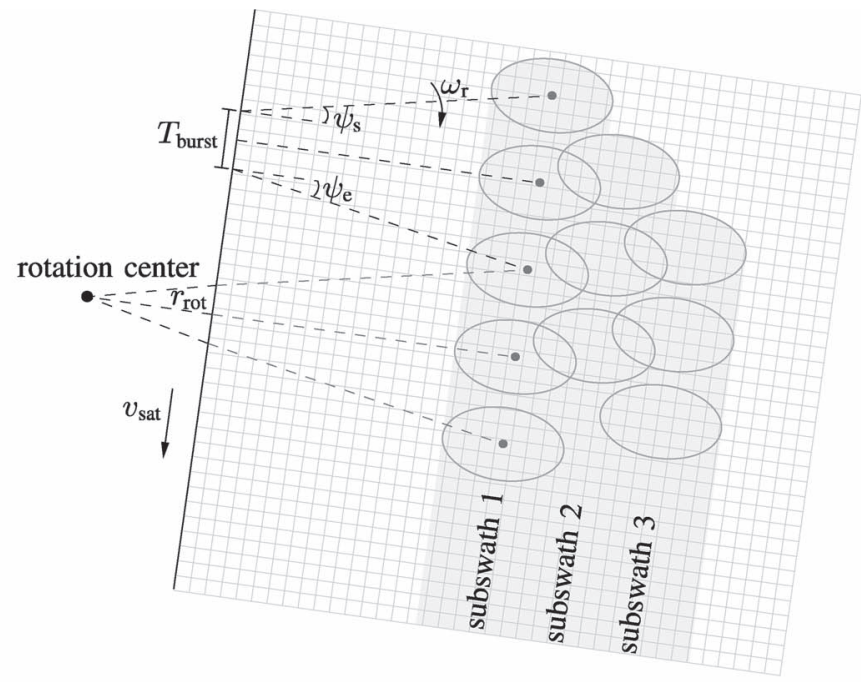

(b)

Fig. 1. Sketch of the acquisition geometry of the two modes. $\psi_{s}$ and $\psi_{e}$ are the start and end squint angles, respectively, $v_{\text {sat }}$ is the forward velocity of the satellite, $r_{\text {rot }}$ is the rotation range distance, and $T_{\text {burst }}$ is the burst duration, which only applies to TOPS. The rotation center in sliding spotlight is further away from the swath, while, in the TOPS mode, it lies behind the sensor. (a) Sliding spotlight. (b) TOPS.

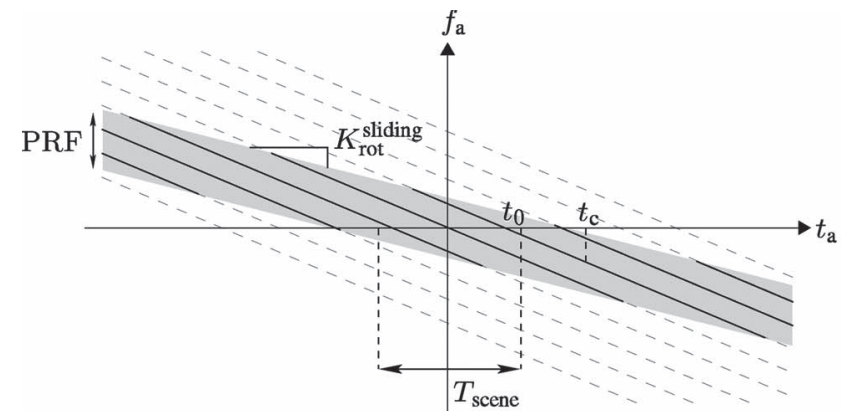

(a)

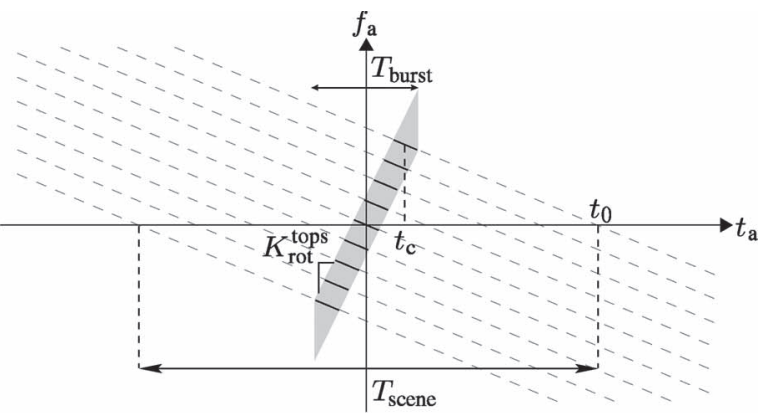

(b)

Fig. 2. TFDs of the two modes. The solid lines represent the targets observed within the main antenna beam (the gray area represents the instantaneous azimuth bandwidth). $t_{a}$ is the azimuth time, $f_{a}$ is the azimuth frequency, $t_{0}$ is the zero-Doppler time, and $t_{c}$ is the beam-center time, i.e., the time instant when the target is illuminated by the maximum of the antenna pattern. The slope of the gray area is given by the Doppler rate at the rotation center. (a) Sliding spotlight. (b) TOPS (one burst).

consequently, the resolution is improved but at the expense of scene extension along the azimuth dimension. A compromise between staring spotlight and stripmap is given by the sliding spotlight mode [11], [12], where the rotation center of the antenna beam is located beyond the swath, hence increasing the observed azimuth scene extension at the expense of azimuth resolution. Figs. 1(a) and 2(a) show the acquisition geometry and the time-frequency diagram (TFD) for the sliding spotlight mode, respectively. Note that the total azimuth bandwidth is greater than the PRF (gray area at a given time instant).

\section{B. TOPS}

TOPS has been proposed as a new wide-swath imaging mode [1]. It overcomes the problems of scalloping and azimuthvarying signal-to-ambiguity ratio of the conventional ScanSAR mode by means of steering the antenna in the along-track direction. To achieve the same swath coverage and avoid the undesired effects of ScanSAR, the antenna is rotated throughout the acquisition from backward to forward at a constant rotation rate $\omega_{r}$ [see Fig. 1(b)], opposite to the spotlight case. The fast steering leads to a reduction in the observation time and, consequently, a worsening of the azimuth resolution. However, now, all targets are observed by the complete azimuth antenna pattern, and, therefore, the scalloping effect disappears, and azimuth ambiguities and signal-to-noise ratio become constant in the azimuth dimension. At the end of the burst, the antenna look angle is changed to illuminate a second subswath, pointing again backwards. When the last subswath is imaged, the antenna points back to the first subswath so that no gaps are left between the bursts of the same subswath.

Fig. 2(b) shows the TFD of one TOPS burst. The total azimuth bandwidth spans several PRF intervals, as in the spotlight case. Note also that the rotation center is located behind the sensor, and, as it happens in the ScanSAR mode, the focused burst is much larger than the raw data burst, requiring special care when performing the azimuth focusing.

A second possibility, called inverse TOPS [3], is to steer the antenna in the same direction as spotlight using a rotation center placed between the sensor and the illuminated swath. In theory, the same performance as normal TOPS can be achieved, and the processing is the same as for the conventional TOPS. 


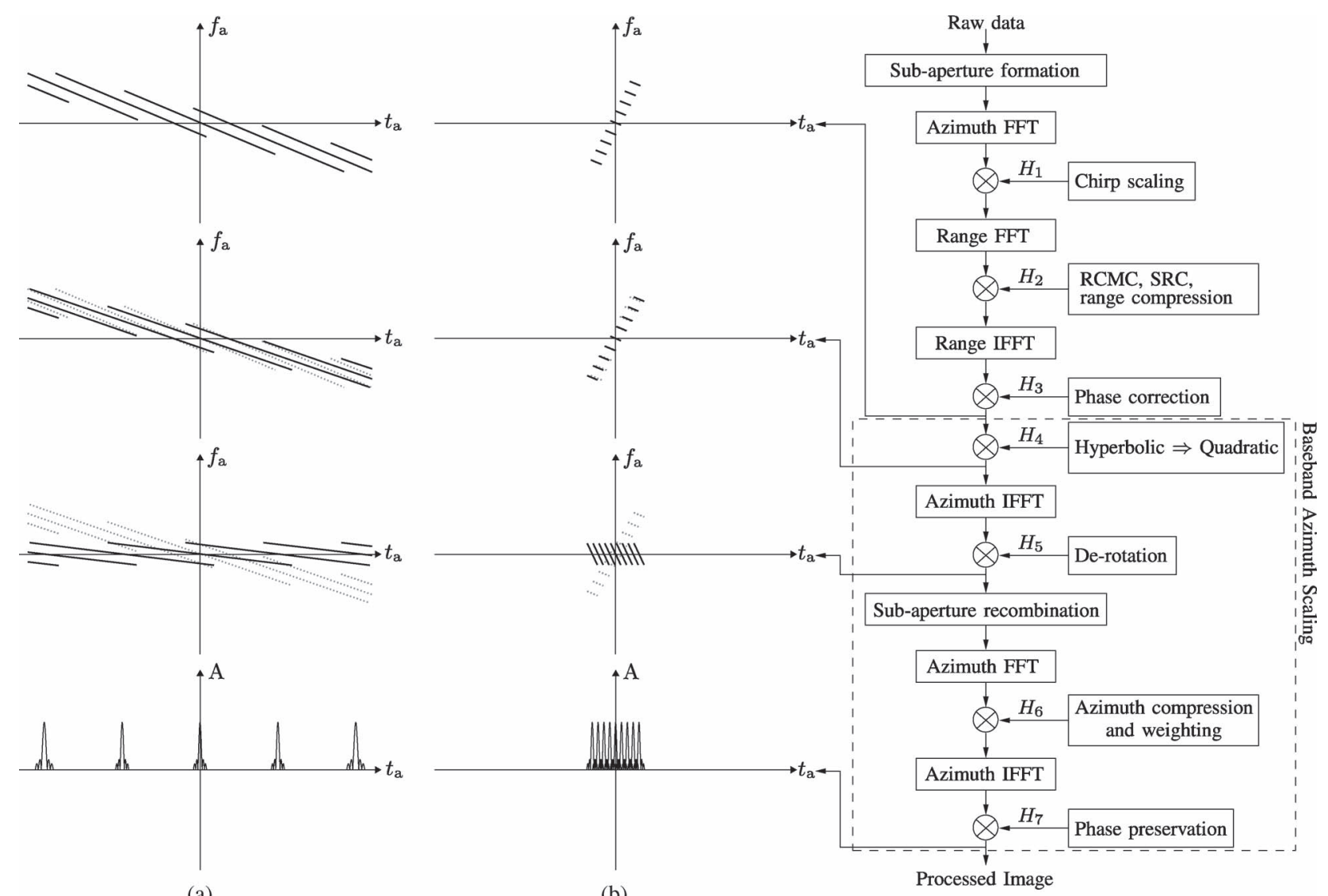

Fig. 3. Block diagram of the proposed processor for sliding spotlight and TOPS imaging modes. The first three rows of the pictures on the left show the TFDs before and after each BAS step for both sliding spotlight and TOPS imaging modes, where the time and frequency spans are the same. In the second and third row, the dotted gray and solid black lines represent the signals of the different targets before and after the corresponding step, respectively. The targets are located at the same range distance but at different azimuth positions. In both modes, the scaling range is not equal to the range of the targets. The last row shows the time-amplitude diagram of the focused signal. The new part of the processor is the BAS, including the phase functions $H_{4}-H_{7}$. (a) Sliding spotlight. (b) TOPS (one burst).

\section{BAS}

\section{A. Theoretical Formulation}

The block diagram of the proposed processor appears in Fig. 3, while the Appendix derives the signal after each step of BAS. In order to accommodate the larger scene bandwidth in the sliding spotlight and TOPS modes, the data are divided in azimuth blocks (subapertures), similar to the approach in [7]. The subaperture size is selected, taking advantage of the higher PRF with respect to the processed azimuth bandwidth $B_{a \text {,proc. }}$ The size in seconds of the subaperture can be computed with

$$
T_{\mathrm{sub}}=\frac{\mathrm{PRF}-B_{a, \mathrm{proc}}}{\left|K_{\mathrm{rot}}\right|}
$$

where $K_{\text {rot }}$ is the slope of the varying Doppler centroid introduced by the rotation of the antenna (see Fig. 2). Using the subaperture size given by (1), no aliasing is introduced into the final focused image. After the division into subapertures, the processing for each subaperture is continued with the corresponding Doppler centroid. To achieve a smooth subaperture recombination, the subapertures are formed with some overlap, e.g., $5 \%$. The steps of RCM correction, secondary range compression, and range compression are carried out using the standard phase functions of Extended Chirp Scaling (ECS) [6] (functions $H_{1}, H_{2}$, and $H_{3}$ ) for each subaperture, but note that, for this purpose, other SAR processing kernels can also be used. Once in the range-Doppler domain, BAS is performed, which consists of the phase functions $H_{4}, H_{5}, H_{6}$, and $H_{7}$. The first step is the removal of the hyperbolic azimuth phase and its replacement with a purely quadratic phase shape using

$$
\begin{aligned}
H_{4}\left(f_{a}, r\right)= & \exp \left[j \frac{4 \pi}{\lambda} r \cdot\left(\beta\left(f_{a}, r\right)-1\right)\right] \\
& \cdot \exp \left[-j 2 \pi f_{a} t_{v}(r)\right] \cdot \exp \left[-j \frac{\pi}{K_{\mathrm{scl}}(r)} f_{a}^{2}\right]
\end{aligned}
$$

where $f_{a}$ is the azimuth frequency, $r$ is the range vector of the closest approach distances, $\lambda$ is the wavelength, and

$$
\beta\left(f_{a}, r\right)=\sqrt{1-\left(\frac{\lambda f_{a}}{2 v_{\text {eff }}(r)}\right)^{2}}
$$

where $v_{\text {eff }}(r)$ is the range-dependent effective velocity. The assumption of a hyperbolic phase history made by (3) needs to be checked for every particular satellite system, as this assumption becomes less accurate the larger the observation time. Whenever this assumption were not valid, a numerical evaluation of 
(3) as presented in [13]-[15] would be required. Note that the hyperbolic approximation is valid for the TerraSAR-X case.

The quadratic phase history inserted in (2) is described by the scaling Doppler rate

$$
K_{\mathrm{scl}}=-\frac{2 v_{\mathrm{eff}}^{2}\left(r_{\mathrm{mid}}\right)}{\lambda r_{\mathrm{scl}}(r)}
$$

where $v_{\text {eff }}\left(r_{\text {mid }}\right)$ is the effective velocity at midrange. The scaling range $r_{\mathrm{scl}}(r)$ is a function of $r$ (it is neither constant as in [6] nor equal to the original range vector $r$ as in [16]). Its expression is given in Section III-B. Since $H_{4}$ leads to a shift of the azimuth signals that are not located at the scaling range, a slight extension of the azimuth time dimension is required. However, this extension usually falls within the range attained by the extension of the subaperture size to the next power of two. Nevertheless, in order to minimize the needed extension, a linear phase ramp is introduced, which is given by the second exponential term in (2). The time shift $t_{v}(r)$, whose expression can be found in [7], causes the required time extension to be symmetric.

In the next step, an azimuth inverse fast Fourier transform (FFT) is used for a transformation back to the azimuth/range time. However, the total azimuth bandwidth of all the subapertures still exceeds the PRF. Therefore, a demodulation is carried out that is similar as that in [1] and [9] by using the following derotation function:

$$
H_{5}\left(t_{a}, r\right)=\exp \left[-j \pi K_{\text {rot }}(r) \cdot\left(t_{a}-t_{\text {mid }}\right)^{2}\right]
$$

where $t_{a}$ is the azimuth time and $t_{\text {mid }}$ is the scene center time. The chirp rate used in the derotation function depends on the range and is given by [see Section III-B for the definition of $\left.r_{\text {rot }}(r)\right]$

$$
K_{\text {rot }}(r)=-\frac{2 v_{\text {eff }}^{2}\left(r_{\text {mid }}\right)}{\lambda r_{\text {rot }}(r)} .
$$

Next, the individual subapertures are assembled. The effect on the signal due to the derotation function can be seen in Fig. 3 for both imaging modes. At this point, the effective chirp rate of the signal has been changed to $K_{\mathrm{eff}}(r)=K_{\mathrm{scl}}(r)-K_{\mathrm{rot}}(r)$. Due to the fact that, now, the data spectrum for all the targets is demodulated, matched filtering can be applied using

$$
H_{6}\left(f_{a}, r\right)=W\left(f_{a}\right) \cdot \exp \left[j \frac{\pi}{K_{\mathrm{eff}}(r)} f_{a}^{2}\right]
$$

with

$$
-\mathrm{PRF}+f_{\mathrm{dc}}<f_{a}<\mathrm{PRF}+f_{\mathrm{dc}}
$$

where $f_{\mathrm{dc}}$ is the mean Doppler centroid of the data acquisition. Note that azimuth sidelobe suppression can also be performed at this stage by means of a weighting function $W\left(f_{a}\right)$. An inverse FFT results in a focused signal. However, for phase preserving processing, the data must be multiplied by the following phase function:

$$
H_{7}\left(t_{a}, r\right)=\exp \left[j \pi K_{t}(r) \cdot\left(1-\frac{r_{\mathrm{scl0}}}{r_{\text {rot0 }}}\right)^{2} \cdot\left(t_{a}-t_{\text {mid }}\right)^{2}\right]
$$

where

$$
K_{t}(r)=-\frac{2 v_{\mathrm{eff}}^{2}\left(r_{\mathrm{mid}}\right)}{\lambda \cdot\left(r_{\mathrm{rot}}(r)-r_{\mathrm{scl}}(r)\right)}
$$

and the definition of $r_{\text {rot0 } 0}$ and $r_{\mathrm{scl} 0}$ is given in the next section.

The proposed processor achieves the phase preserving focusing in an efficient way without interpolations. Furthermore, the azimuth image sampling can be selected as shown in the following section, which is of interest in the TOPS mode to avoid interpolations when combining the subswaths.

\section{B. Selection of the Scaling and Rotation Vectors}

In order to obtain the scaling and rotation range vectors, one has to take into account two facts. First, note that the azimuth image sampling after BAS is given by (see the Appendix)

$$
\Delta x_{\text {final }}=\Delta x_{\text {orig }} \cdot\left(1-\frac{r_{\text {scl }}}{r_{\text {rot }}}\right)
$$

where $r_{\mathrm{scl}}$ is the scaling range used in $H_{4}, r_{\text {rot }}$ is the rotation distance used in $H_{5}$, and $\Delta x_{\text {orig }}$ is the original sampling that is equal to $v_{g} / \mathrm{PRF}$, where $v_{g}$ is the ground velocity. On the other hand, the scaling operation $\left(H_{4}\right)$ modifies the rotation range, as depicted in Fig. 3 and expressed by

$$
r_{\text {rot }}(r)=r_{\text {rot } 0}-\left(r-r_{\text {scl }}\right)
$$

where $r_{\text {rot0 }}$ is the vector distance to the rotation center given by the geometry, and note that no change occurs when the scaling range is equal to the range of the target. If a constant scaling range is used, a constant rotation range must be selected, as, otherwise, the azimuth sampling would be range dependent as shown in (11). However, because the effective rotation range changes as given by (12), not all targets would be at the baseband in that case, hence resulting in aliasing. Therefore, as it is desired to have the same azimuth image sampling for all the ranges and, at the same time, properly demodulate all the targets, the combination of (11) and (12) yields the following scaling and rotation vectors:

$$
\begin{aligned}
& r_{\mathrm{scl}}(r)=\frac{r_{\mathrm{scl} 0}}{r_{\text {rot } 0}} r_{\text {rot }}(r) \\
& r_{\text {rot }}(r)=\frac{r_{\text {rot } 0}-r}{1-r_{\text {scl0 }} / r_{\text {rot } 0}}
\end{aligned}
$$

where $r_{\mathrm{scl} 0}$ is a scaling range selected considering the desired azimuth image sampling and should be within the range of the respective subswath in order to minimize the needed extension of the subapertures. By doing so, the azimuth image sampling is given by

$$
\Delta x_{\text {final }}=\Delta x_{\text {orig }} \cdot\left(1-\frac{r_{\text {scl0 }}}{r_{\text {rot0 }}}\right) .
$$

Note that, in the TOPS mode, $r_{\text {roto }}$ is negative so that the new image sampling is larger than the original raw data sampling. This is desirable, as the resolution is worse than in the stripmap case. 


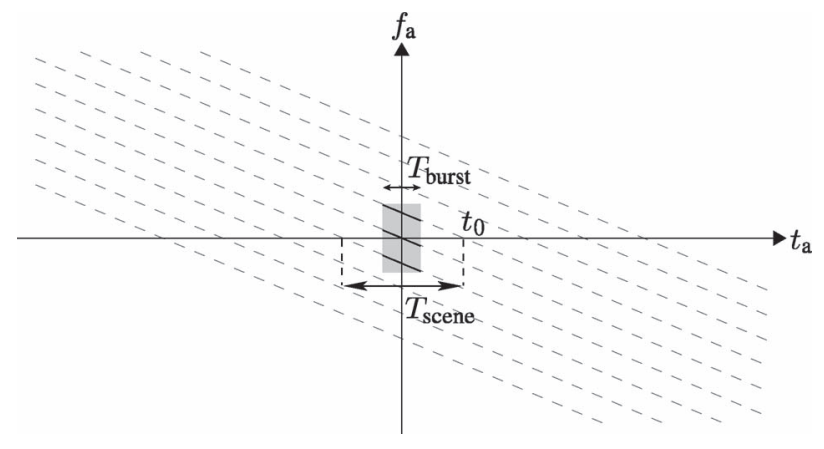

Fig. 4. TFD of the ScanSAR mode. The time and frequency spans are the same as those shown in Fig. 2.

\section{Processing of ScanSAR Data With BAS}

The ScanSAR mode is a well-known wide-swath imaging mode [5], [6], [8], [17], [18], which extends the illuminated swath by periodically switching the antenna pointing to illuminate different subswaths. Therefore, the azimuth resolution is worse when compared to the stripmap case due to the smaller illumination time. Fig. 4 shows the TFD of one burst, which has the same time and frequency spans as those shown in Fig. 2. It can be observed how the Doppler centroid varies depending on the azimuth position of the target, but, opposite to the spotlight and TOPS cases, the total azimuth bandwidth lies within the PRF. Similar as TOPS, the scene extension is larger than the raw data burst, hence requiring special attention when performing the azimuth focusing. There exist several approaches to solve this problem in an efficient way [5], [6], [8]. Indeed, BAS can also process the ScanSAR data. The solution is less efficient than some of the existing approaches but still more efficient than the standard matched filtering approach, which requires a large time extension to avoid folding in time.

ScanSAR is a special case concerning BAS, as no explicit rotation range exists. However, one can profit of the fact that changing the Doppler rate shifts the signals by an amount that is equal to

$$
\delta t\left(t_{a}, r\right)=\left(r_{\mathrm{scl}}-r\right) \frac{\tan \psi\left(t_{a}\right)}{v_{g}}
$$

where $\psi\left(t_{a}\right)$ is the squint angle at time instant $t_{a}$. Since $\psi\left(t_{a}\right)$ depends on the azimuth position of the target in the ScanSAR case, this step introduces an artificial rotation range, which can be used to derotate the azimuth spectrum and focus the signal using BAS. Fig. 5 shows this effect. Note, again, that the derotation shall depend also on the range, as the quotient between the scaling and rotation vectors must have the same value for every range. Consequently, the equations to obtain the scaling and rotation vectors in ScanSAR are

$$
\begin{aligned}
& r_{\mathrm{scl}}^{\mathrm{scan}}(r)=r \cdot(1-\alpha) \\
& r_{\mathrm{rot}}^{\mathrm{scan}}(r)=r_{\mathrm{scl}}^{\mathrm{scan}}(r)-r=-\alpha r
\end{aligned}
$$

where $\alpha$ is a value between zero and one and can be selected as a function of the desired azimuth image sampling, and (12) has

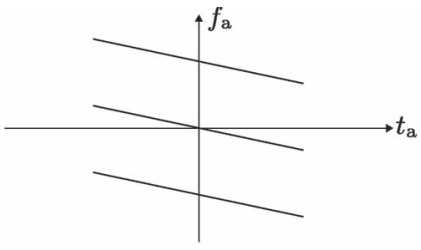

(a)

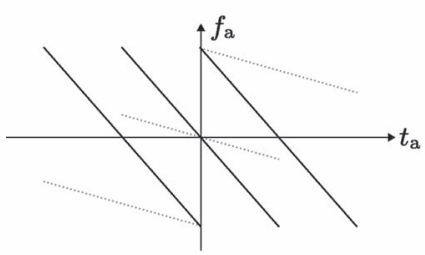

(c)

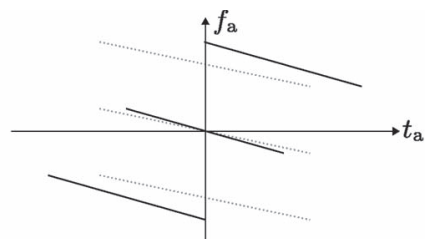

(b)

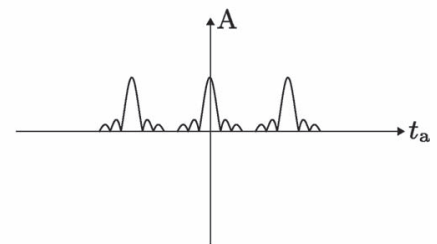

(d)
Fig. 5. Diagrams of a ScanSAR burst before and after each step of BAS. The three targets are located at the same range bin but different azimuth positions. (a) TFD before $H_{4}$. (b) TFD after $H_{4}$. (c) TFD after $H_{5}$. (d) Time-amplitude diagram after $\mathrm{H}_{6}$ and $\mathrm{H}_{7}$.

been used with $r_{\text {rot }}=0$ to obtain (18). The final pixel spacing is then given by

$$
\Delta x_{\text {final }}^{\text {scan }}=\frac{\Delta x_{\text {orig }}}{\alpha}
$$

where $\alpha$ must be large enough so that the final sampling accomplishes the Nyquist criterion for the given ScanSAR resolution. Note that $r_{\mathrm{scl}}(r)$ is never equal to $r$ as that would mean that no shift of the signal in time is occurring for that particular range. Consequently, no artificial $r_{\text {rot }}$ would be introduced, preventing the use of BAS as such. This can represent a drawback if efficiency is considered, as the extension to accommodate the shift of the signal due to $H_{4}$ will be larger than that in the sliding spotlight and TOPS modes. The necessary extension in the ScanSAR case is given by [6]

$$
T_{\text {ext }}=T_{a} \cdot|\alpha|-T_{\text {burst }} \cdot \alpha
$$

where $T_{\text {burst }}$ is the burst duration and $T_{a}$ corresponds to the footprint of the antenna, i.e., the stripmap synthetic aperture length in seconds. The first term in (20) accounts for the shift described in (16), while the second term considers the shrinking or stretching of the signal envelope. As an example, consider a ScanSAR system with $T_{\text {burst }}=T_{a} / 4$, and assuming $\alpha=$ 0.25 yields an extension that is equal to $0.75 \cdot T_{\text {burst }}$, i.e., the time extension is almost the same length as the burst itself. Nevertheless, it is not three times more, as it would be required in order to use the standard matched filtering, noting also that, in the latter, the signal would be unnecessarily oversampled.

Finally, note that, in (9), the term $\left(1-r_{\mathrm{scl} 0} / r_{\text {roto }}\right)^{2}$ should be substituted by $1 / \alpha^{2}$ in the ScanSAR case, as no explicit definitions for $r_{\mathrm{scl} 0}$ nor $r_{\text {rot0 } 0}$ exist. Furthermore, the subaperture formation and recombination are not necessary to process a burst in ScanSAR, as the azimuth bandwidth falls within the system PRF.

\section{Discussion on Staring Spotlight}

The staring spotlight mode is a particular realization of the sliding spotlight mode when the rotation range is at the scene 


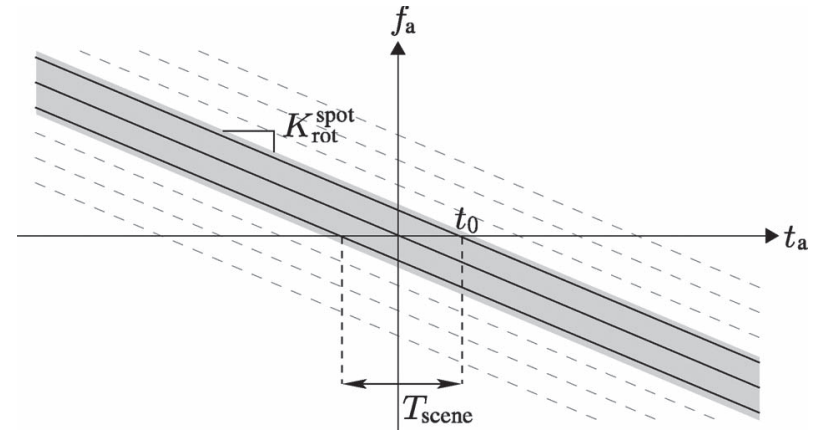

Fig. 6. TFD of the staring spotlight mode. Note that the rotation center is at the scene center. The time and frequency spans are the same as those shown in Fig. 2.

center, as depicted in Fig. 6. This configuration prevents the use of the proposed BAS approach because, after derotation, the targets have no modulation left, i.e., the effective chirp rate $K_{\text {eff }}$ is zero. This is, indeed, the approach in [7], where a subsequent FFT already focuses the data in the frequency domain (SPECAN approach). It might be possible to leave a small effective chirp rate in the signal, but, then, the targets at the scene edges would not be properly demodulated. Furthermore, even assuming a PRF that is larger than the azimuth bandwidth, the processing would not be efficient due to the needed extension to avoid folding in time. Therefore, BAS is not suitable for staring spotlight.

At this point, it is interesting to find out the limits of BAS when processing sliding spotlight data, i.e., which should be the minimum rotation range in order to efficiently apply BAS. First, it must be satisfied that $K_{\text {eff }}$ is nonzero and, second, that the bandwidth of the demodulated spectrum is smaller but close to the instantaneous bandwidth, i.e., the stripmap bandwidth. The bandwidth of the demodulated spectrum is (see Appendix)

$$
B_{w}=\left|K_{\mathrm{eff}} \frac{r_{\mathrm{scl}}}{r_{0}} T_{\mathrm{obs}}\right|
$$

where $T_{\text {obs }}$ is the target observation time, which can be computed as a function of the desired azimuth resolution $\delta_{\mathrm{az}}$ with

$$
T_{\mathrm{obs}}=\frac{\lambda r_{0}}{2 v_{s} \delta_{\mathrm{az}}}
$$

where $v_{s}$ is the satellite velocity. By taking the instantaneous bandwidth as

$$
B_{w}^{\mathrm{inst}} \approx \frac{2 v_{s} \theta_{\mathrm{az}}}{\lambda}
$$

where $\theta_{\mathrm{az}}$ is the $3-\mathrm{dB}$ azimuth antenna beamwidth, the required rotation range must satisfy

$$
r_{\mathrm{rot} 0} \geq \frac{r_{\mathrm{scl}}}{1-\gamma \frac{r_{0} \theta_{\mathrm{az}}}{v_{g} T_{\mathrm{obs}}}}
$$

where $0<\gamma<1$ specifies how much of the instantaneous bandwidth should be occupied by the demodulated spectrum. A conservative threshold would be $\gamma \geq 0$.8. If the threshold is relaxed, it might occur that the image sampling is much
TABLE I

Main System and Processing Parameters FOR THE SiMULATION IN SECTION IV-A

\begin{tabular}{lc}
\hline Carrier frequency & $9.65 \mathrm{GHz}$ \\
Azimuth beamwidth $(3 \mathrm{~dB})$ & $0.33^{\circ}$ \\
System PRF & $3475 \mathrm{~Hz}$ \\
Sampling frequency & $150 \mathrm{MHz}$ \\
Height & $514 \mathrm{~km}$ \\
Effective velocity & $6800 \mathrm{~m} / \mathrm{s}$ \\
Mean look angle & $30.42^{\circ}$ \\
Maximum steering angle & $\pm 0.43^{\circ}$ \\
Burst duration & $0.2667 \mathrm{~s}$ \\
Imposed azimuth resolution & $16 \mathrm{~m}$ \\
Rotation range & $-120.8 \mathrm{~km}$ \\
Scaling range & $596.1 \mathrm{~km}$ \\
Image sampling (range $\times$ azimuth) & $1.0 \mathrm{~m} \times 11.61 \mathrm{~m}$ \\
\hline
\end{tabular}

smaller than the image resolution, hence requiring an extension in the time domain before the last forward azimuth FFT to avoid folding in time.

This same effect can also be explained by considering the scaling operation, which, in the sliding spotlight case, stretches the azimuth time axis by a factor that is equal to

$$
\alpha=\frac{r_{\text {rot0 }}}{r_{\text {rot0 }}-r_{\mathrm{scl} 0}} .
$$

A target with zero-Doppler position $t_{0}=t_{\text {mid }}+\Delta t$ will appear focused at position $t_{0}^{\prime}=t_{\text {mid }}+\alpha \Delta t$. Folding in time will occur whenever $t_{0}^{\prime}$ lies beyond half of the acquisition time, requiring an extension in the time domain and, hence, reducing the efficiency of the proposed algorithm.

In any case, it is interesting to comment the modifications in the block diagram in Fig. 3 to include the approach presented in [7] in order to process the staring spotlight data. The rangevariant processing is the same, and only the phase functions $H_{4}$, $H_{5}$, and $H_{7}$ are slightly modified, while $H_{6}$ and the last inverse azimuth FFT are not needed. Therefore, $H_{4}$ is given by

$$
\begin{aligned}
H_{4}^{\mathrm{ss}}\left(f_{a}, r\right)=\exp & {\left[j \frac{4 \pi}{\lambda} r \cdot\left(\beta\left(f_{a}, r\right)-1\right)\right] } \\
\cdot & \exp \left[-j 2 \pi f_{a} t_{v}(r)\right] \cdot \exp \left[-j \frac{\pi}{K_{\mathrm{scl}}^{\mathrm{ss}}} f_{a}^{2}\right]
\end{aligned}
$$

where the superscript $s s$ stands for staring spotlight. Note that a constant scaling range for all the ranges is used. Deramping is then performed with the following 1-D phase function:

$$
H_{5}^{\mathrm{ss}}\left(t_{a}\right)=W\left(t_{a}\right) \cdot \exp \left[-j \pi K_{\mathrm{scl}}^{\mathrm{ss}} \cdot\left(t_{a}-t_{\mathrm{mid}}\right)^{2}\right]
$$

where $W\left(t_{a}\right)$ is the weighting function, which can efficiently be applied in the time domain in the staring spotlight mode. A subsequent azimuth FFT focuses the data in the Fourier domain, where every target is focused at a frequency given by

$$
f_{0}=K_{\mathrm{scl}}^{\mathrm{ss}} \cdot\left(t_{0}-t_{\mathrm{mid}}\right)
$$

so that, similar to BAS, the scaling range is selected as a function of the desired azimuth image sampling. Finally, 

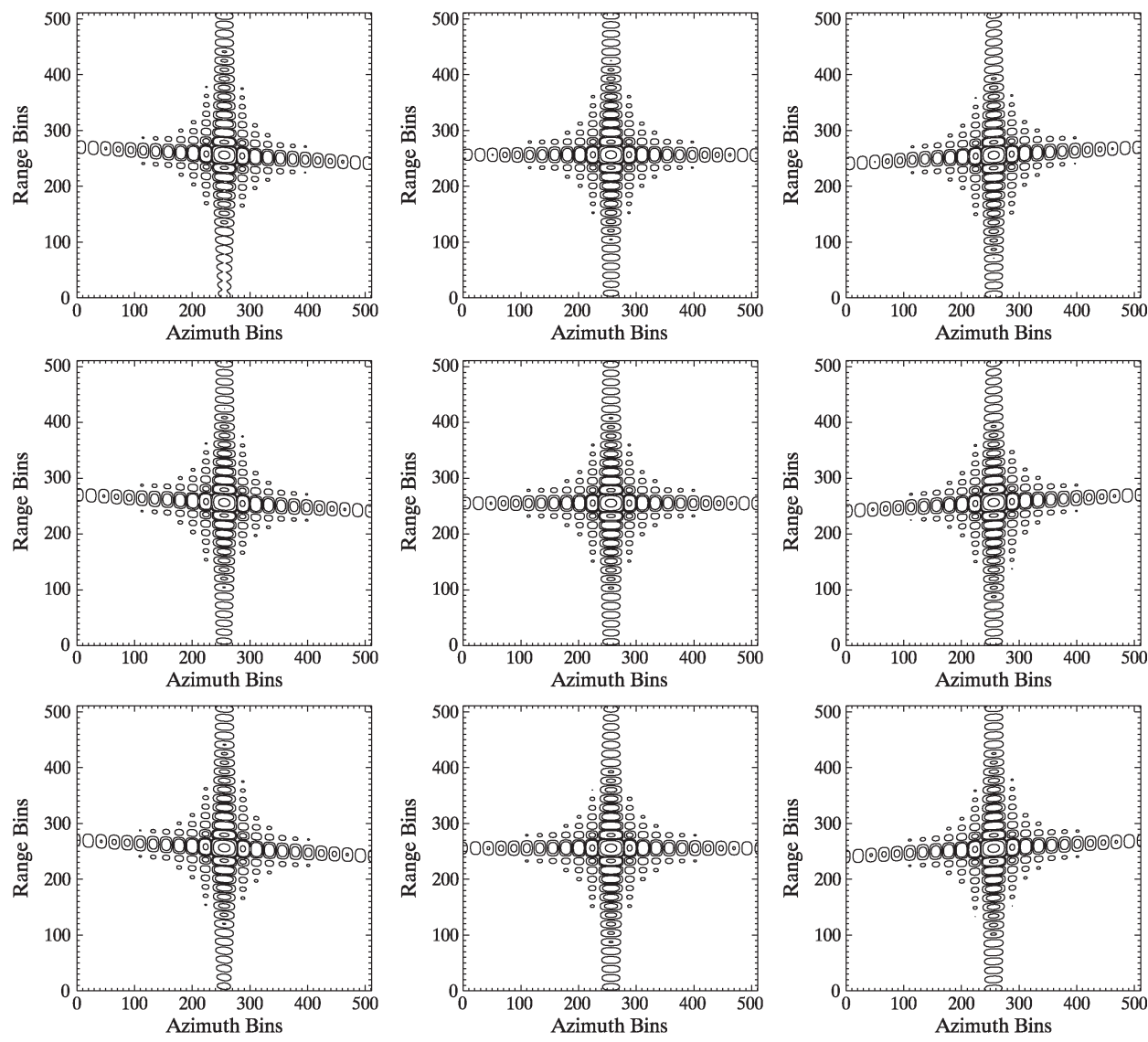

Fig. 7. Contour plots showing the processing result of nine point targets located at (top) near, (middle) mid-, and (bottom) far range within a TOPS burst. Contour lines at $-3,-15,-30$, and $-40 \mathrm{~dB}$.

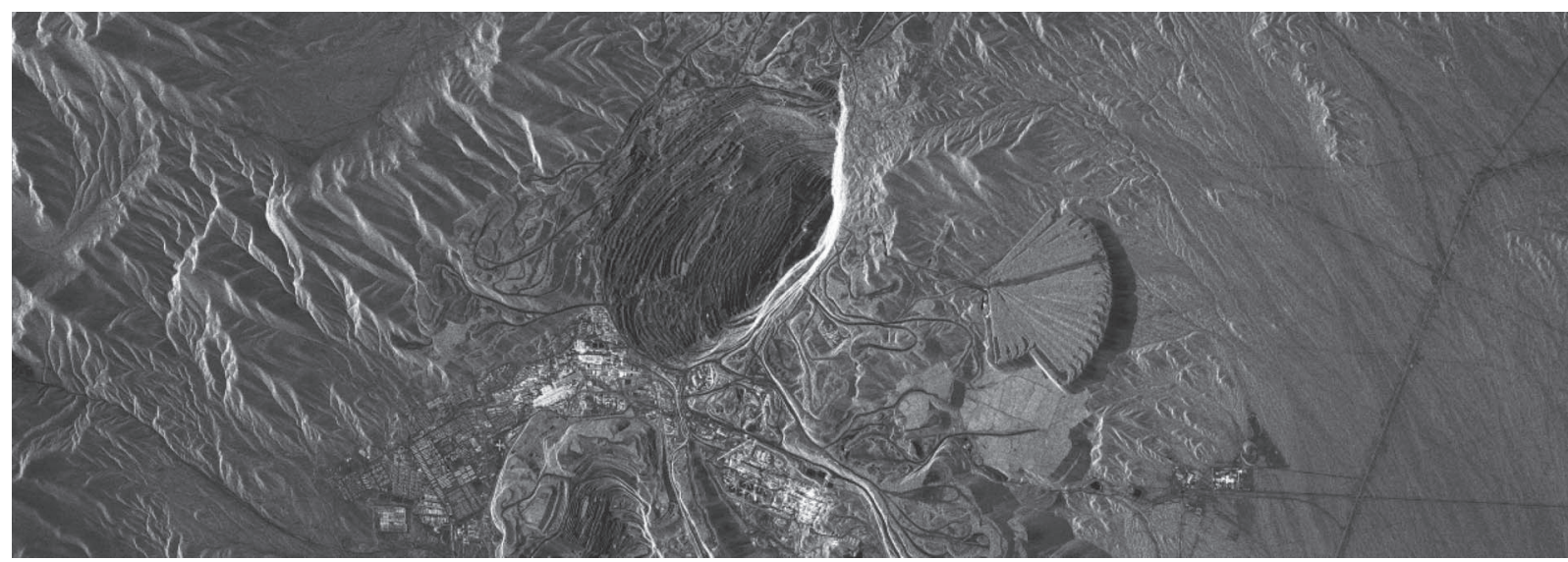

Fig. 8. Sliding spotlight image acquired by TerraSAR-X over the Chuquicamata copper mine, Chile. The range is horizontal, and the azimuth is vertical, with near range on the left side. The image dimensions are $6.8 \mathrm{~km} \times 10.9 \mathrm{~km}$ (azimuth $\times$ slant range).

phase preservation is achieved with the following phase multiplication:

$$
H_{7}^{\mathrm{ss}}\left(f_{a}\right)=\exp \left[j \pi \frac{f_{a}^{2}}{K_{\mathrm{scl}}^{\mathrm{ss}}}\right] .
$$

Therefore, with the necessary adjustments, the proposed block diagram can be used to process the data in four acquisition modes other than stripmap, namely, sliding and staring spotlight, TOPS, and ScanSAR. The azimuth processing can be performed either with BAS (sliding spotlight, TOPS, and
ScanSAR) or with SPECAN and standard azimuth scaling, i.e., the approach initially proposed for staring spotlight [7], sliding spotlight [12], and ScanSAR [6].

\section{E. Discussion}

An interesting observation with BAS is that the image sampling is changed drastically. For example, in the TOPS mode using the system parameters of TerraSAR-X shown in Table I, the final azimuth sampling is about four times the original one. It occurs similarly with the other modes. Indeed, as stated in its 
name, BAS is nothing but a scaling approach as used in ECS to perform RCM equalization [6], [19] or as in [16] to change the azimuth image sampling. In such cases, the scaling operation is applied to the demodulated data. The main limitation when applying the scaling approach is that, if the new sampling is too different from the original one, aliasing in the frequency domain might be introduced [6]. However, in the imaging modes under study, aliasing is what one is looking for, i.e., a large shift of the spectrum is introduced in order to demodulate it. Therefore, every phase function of BAS has a clear interpretation linked to the different steps to perform compression, but an interpretation as a classical scaling approach is also possible. The key point in BAS is the selection of the different scaling and rotation ranges in order to properly demodulate all the targets and, at the same time, maintain the same image sampling for all the ranges.

\section{EXPERIMENTAL RESULTS}

\section{A. Simulation Results}

In order to validate the proposed BAS approach, the TOPS raw data of nine point targets were simulated. The main system and processing parameters appear in Table I. Fig. 7 shows the interpolated contour plot of the processed targets. The nine point targets have different range and azimuth positions corresponding to the same TOPS burst. They are arranged in such a way that the targets in the corner positions limit a scene with the dimensions of $7.2 \mathrm{~km} \times 24.6 \mathrm{~km}$ in the azimuth and range, respectively. The maximum deviations of the measured resolutions from the theoretical values are $2 \%$ in the azimuth and $1 \%$ in the range. The phase preservation of the algorithm was also validated and was found to be within $1^{\circ}$ accuracy. The same simulations were carried out with sliding spotlight and ScanSAR, with similar results.

\section{B. Results With TerraSAR-X Data}

The real data acquired by TerraSAR-X in TOPS and sliding spotlight modes are presented next. Fig. 8 shows a sliding spotlight image processed with the proposed SAR processor using the BAS algorithm. The main system and processing parameters appear in Table II. The data were acquired on July 4, 2007, over the Chuquicamata copper mine, located in the Atacama Desert, Chile. A Hamming window was applied in both dimensions for sidelobe suppression.

The flexibility of the phased array antenna of TerraSAR-X concerning the operational commanding has allowed an efficient implementation of the TOPS mode even though this mode was not foreseen when designing the system. A data take over Uyuni salt lake, Bolivia, was acquired on September 29, 2007, in a descending orbit configuration. The main system and processing parameters of the data take appear in Table III. The TOPS acquisition consists of four subswaths, with a commanded resolution of $16 \mathrm{~m}$. The azimuth image sampling that minimizes the extension considering all the subswaths is $9.2 \mathrm{~m}$. Fig. 9 shows the focused image, with scene dimensions of $120.7 \mathrm{~km} \times 68.1 \mathrm{~km}$ (azimuth $\times$ slant range). There are a total of eight bursts per subswath, and the anticipated absence of scalloping is evident [3].

The image quality parameters have been analyzed as well as the phase preservation using an interferometric pair of the data take shown in Fig. 9. All the results are well in accordance with the theoretical and simulated values [20].

\section{CONCLUSiON}

This paper has presented a new azimuth scaling approach, named BAS, which is suitable to process the data acquired in sliding spotlight and TOPS imaging modes. It achieves an efficient focusing of the azimuth signal by selecting an image sampling that is close to its resolution. The azimuth-dependent Doppler centroid due to the steering of the antenna permits such a drastic change of the image sampling with BAS, which would lead to aliasing in a conventional stripmap signal. The whole processing algorithm has been presented. It includes a subaperture approach to take into account the total azimuth bandwidth, which can span over several PRF intervals. Any kernel can be selected to perform the range-variant processing, but, here, the so-called ECS has been proposed [6], with the benefit that the whole processing, including BAS, is performed without interpolations. BAS has also the advantage that the azimuth image sampling can be selected, which is useful in the TOPS case in order to impose the same sampling to all the subswaths, avoiding, again, the need of interpolations when combining them.

It has been further shown that BAS can also be used to process the data acquired in the ScanSAR mode. Although less efficient than some existing approaches, it shares the same kernel as for TOPS and sliding spotlight, with the only difference being the computation of the scaling and rotation range vectors. In any case, it is interesting to have a kernel that is capable of processing three different imaging modes.

A comment has also been made concerning staring spotlight since BAS is not suitable to process the data acquired in this particular mode. Therefore, a criterion has been provided in order to know whether BAS can efficiently process or not a given sliding spotlight acquisition. The necessary modifications in the proposed algorithm to process the staring spotlight data have been also included for completeness.

Finally, the simulated data of nine point targets and the real data acquired by TerraSAR-X in sliding spotlight and TOPS imaging modes have been used to validate the performance of the proposed processor.

\section{APPENDIX}

This appendix is meant to help understand the different steps of BAS. The 1-D signal of one target after RCM correction without unnecessary terms can be expressed as

$$
s_{0}\left(t ; r_{0}\right)=\operatorname{rect}\left(\frac{t-t_{c}}{T_{\mathrm{obs}}}\right) \cdot \exp \left[-j \frac{4 \pi}{\lambda} R\left(t ; r_{0}\right)\right]
$$

where $r_{0}$ is the closest approach range distance, $R\left(t ; r_{0}\right)$ is the distance between the target and the sensor as a function of the 
TABLE II

Main System and Processing Parameters FOR THE SLIDING SPOTLIGHT ACQUISITION

\begin{tabular}{lc}
\hline Carrier frequency & $9.65 \mathrm{GHz}$ \\
Azimuth beamwidth $(3 \mathrm{~dB})$ & $0.33^{\circ}$ \\
PRF & $3798.6 \mathrm{~Hz}$ \\
Sampling frequency & $164.82 \mathrm{MHz}$ \\
Chirp bandwidth & $150 \mathrm{MHz}$ \\
Effective velocity & $7391.42 \mathrm{~m} / \mathrm{s}$ \\
Data take duration & $3.16 \mathrm{~s}$ \\
Azimuth resolution & $1 \mathrm{~m}$ \\
Rotation range & $1061.5 \mathrm{~km}$ \\
Scaling range $\left(=r_{\text {mid }}\right)$ & $617.1 \mathrm{~km}$ \\
Image sampling (range $\times$ azimuth) & $0.91 \mathrm{~m} \times 0.81 \mathrm{~m}$ \\
\hline
\end{tabular}

TABLE III

MAIN SYSTEM AND PROCESSING PARAMETERS FOR THE TOPS ACQUISITION

\section{PRFs}

Sampling frequency

Effective velocity

Maximum steering angles

Burst durations

Azimuth resolution

Rotation ranges

Scaling ranges

Image sampling (range $\times$ azimuth)
Chirp bandwidth

\author{
4391 / 4054 / 3744 / 3437 Hz \\ $109.88 \mathrm{MHz}$ \\ $100 \mathrm{MHz}$ \\ $7373.63 \mathrm{~m} / \mathrm{s}$ \\ $0.67^{\circ} / 0.64^{\circ} / 0.64^{\circ} / 0.62^{\circ}$ \\ $0.48 / 0.47 / 0.49 / 0.48 \mathrm{~s}$ \\ $16 \mathrm{~m}$ \\ $-151 /-155 /-161 /-164 \mathrm{~km}$ \\ 712 / 664 / 623 / $569 \mathrm{~km}$ \\ $1.36 \mathrm{~m} \times 9.21 \mathrm{~m}$
}

azimuth time $t$, and $t_{c}$ is the beam-center position, which is related to the zero-Doppler one with

$$
t_{c}=t_{0}-\frac{r_{0}}{v_{g}} \tan \psi
$$

where $\psi$ is the mean squint angle. After $H_{4}$, the Doppler rate of the signal is changed to that of the scaling range, yielding

$$
\begin{array}{r}
s_{1}\left(t ; r_{0}\right)=\operatorname{rect}\left(\frac{t-t_{c, \mathrm{scl}}}{\frac{r_{\mathrm{scl}}}{r_{0}} T_{\mathrm{obs}}}\right) \cdot \exp \left[j \pi K_{\mathrm{scl}} \cdot\left(t-t_{0}\right)^{2}\right] \\
\cdot \exp \left[-j \frac{4 \pi}{\lambda} r_{0}\right]
\end{array}
$$

where the envelope has been shifted and scaled and, also, the Doppler rate corresponds to the one of the scaling range. After multiplying by $H_{5}$ and rearranging some terms, the signal becomes

$$
\begin{aligned}
s_{2}\left(t ; r_{0}\right)= & \operatorname{rect}\left(\frac{t-t_{c, \mathrm{scl}}}{\frac{r_{\mathrm{scl}}}{r_{0}} T_{\mathrm{obs}}}\right) \cdot \exp \left[j \pi K_{\mathrm{eff}} \cdot\left(t-t_{0}\right)^{2}\right] \\
& \cdot \exp \left[-j \frac{4 \pi}{\lambda} r_{0}\right] \cdot \exp \left[j \pi K_{\mathrm{rot}} \cdot\left(t_{0}^{2}-t_{\mathrm{mid}}^{2}\right)\right] \\
& \cdot \exp \left[-j 2 \pi K_{\mathrm{rot}} \cdot\left(t_{0}-t_{\mathrm{mid}}\right) t\right]
\end{aligned}
$$

where $K_{\text {eff }}=K_{\mathrm{scl}}-K_{\mathrm{rot}}$. The third exponential term is the so-called residual video phase [21], while the last one is responsible for the demodulation of the target. An azimuth FFT

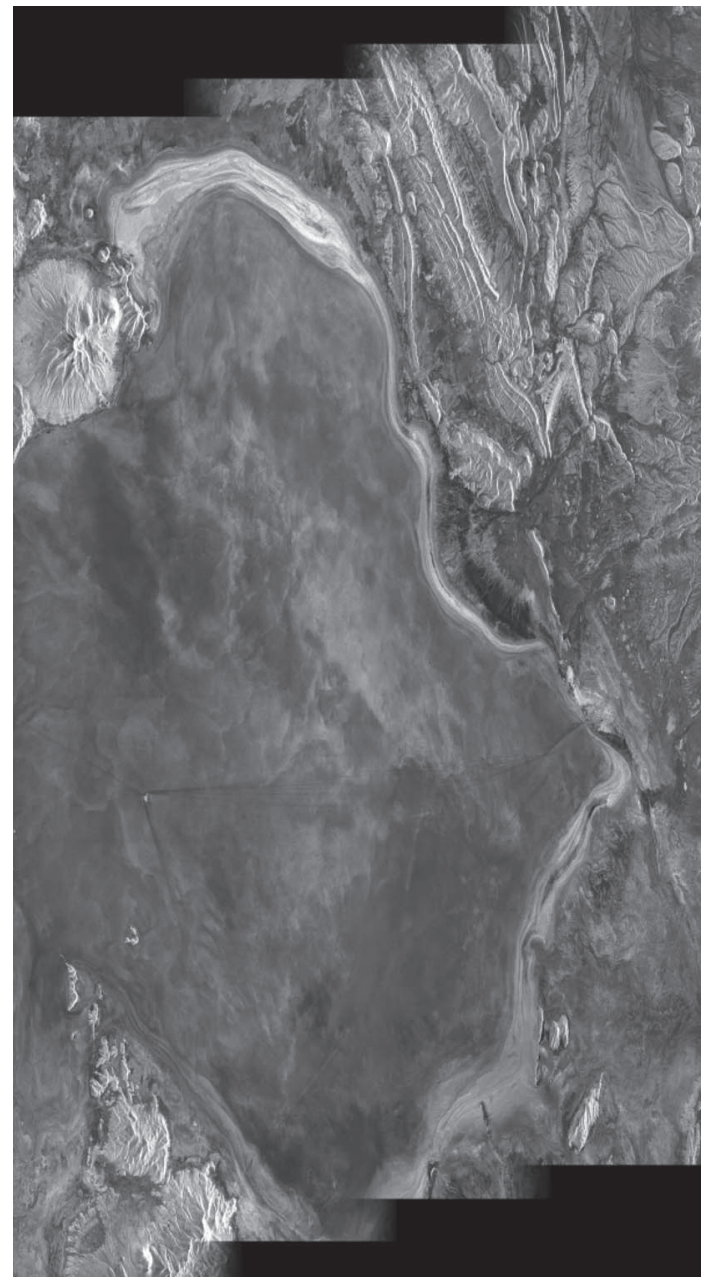

Fig. 9. TOPS image acquired by TerraSAR-X over the Uyuni salt lake, Bolivia. The range is horizontal, and the azimuth is vertical, with near range on the right side. The image dimensions are $120.7 \mathrm{~km} \times 68.1 \mathrm{~km}$ (azimuth $\times$ slant range).

using the stationary phase principle [22] leads to the following signal:

$$
\begin{aligned}
& S_{2}\left(f_{a} ; r_{0}\right) \\
& =\operatorname{rect}\left(\frac{f_{a}}{K_{\mathrm{eff}} \frac{r_{\mathrm{scl}}}{r_{0}} T_{\mathrm{obs}}}\right) \cdot \exp \left[-j 2 \pi f_{a} t_{0}\right] \\
& \quad \cdot \exp \left[-j \frac{\pi}{K_{\mathrm{eff}}}\left(f_{a}+K_{\mathrm{rot}} \cdot\left(t_{0}-t_{\mathrm{mid}}\right)\right)^{2}\right] \cdot \exp \left[-j \frac{4 \pi}{\lambda} r_{0}\right] \\
& \quad \cdot \exp \left[-j \pi K_{\mathrm{rot}} \cdot\left(t_{0}-t_{\mathrm{mid}}\right)^{2}\right] .
\end{aligned}
$$

Finally, after multiplying by $H_{6}$, an inverse FFT leads to

$$
\begin{aligned}
& s_{3}\left(t ; r_{0}\right)=\operatorname{sinc}[ K_{\mathrm{eff}} \frac{r_{\mathrm{scl}}}{r_{0}} T_{\mathrm{obs}} \\
&\left.\cdot\left(t-t_{0}-\frac{r_{\mathrm{scl}}}{r_{\mathrm{rot}}-r_{\mathrm{scl}}} \cdot\left(t_{0}-t_{\mathrm{mid}}\right)\right)\right] \\
& \cdot \exp \left[-j \frac{4 \pi}{\lambda} r_{0}\right] \cdot \exp \left[-j \pi K_{t} \cdot\left(t_{0}-t_{\mathrm{mid}}\right)^{2}\right]
\end{aligned}
$$


where the last exponential term is a phase offset that needs to be corrected and

$$
K_{t}=-\frac{2 v_{\mathrm{eff}}^{2}\left(r_{\mathrm{mid}}\right)}{\lambda \cdot\left(r_{\mathrm{rot}}-r_{\mathrm{scl}}\right)} .
$$

From (35), it can be inferred that the sampling has changed by a factor given by

$$
\Delta x_{\text {new }}=\Delta x_{\text {orig }} \frac{K_{\text {eff }} \frac{r_{\text {scl }}}{r_{0}}}{K_{a}}=\Delta x_{\text {orig }} \cdot\left(1-\frac{r_{\text {scl }}}{r_{\text {rot }}}\right)=\frac{\Delta x_{\text {orig }}}{\alpha}
$$

where $\Delta x_{\text {orig }}=v_{g} / \mathrm{PRF}$ and $K_{a}$ is the original chirp rate of the target. Note also that the new position $t_{0}^{\prime}$ of the target is

$$
t_{0}^{\prime}=\frac{r_{\mathrm{rot}}}{r_{\mathrm{rot}}-r_{\mathrm{scl}}} t_{0}-\frac{r_{\mathrm{scl}}}{r_{\mathrm{rot}}-r_{\mathrm{scl}}} t_{\mathrm{mid}}=t_{\mathrm{mid}}+\alpha \cdot\left(t_{0}-t_{\mathrm{mid}}\right)
$$

which is used to derive $H_{7}$ in order to achieve phase preservation.

\section{REFERENCES}

[1] F. De Zan and A. Monti Guarnieri, "TOPSAR: Terrain observation by progressive scans," IEEE Trans. Geosci. Remote Sens., vol. 44, no. 9, pp. 2352-2360, Sep. 2006.

[2] M. Stangl, R. Werninghaus, B. Schweizer, C. Fischer, M. Brandfass, J. Mittermayer, and H. Breit, "TerraSAR-X technologies and first results," Proc. Inst. Elect. Eng.-Radar Sonar Navig., vol. 153, no. 2, pp. 86-95, Apr. 2006.

[3] A. Meta, P. Prats, U. Steinbrecher, J. Mittermayer, and R. Scheiber, "TerraSAR-X TOPSAR and ScanSAR comparison," in Proc. EUSAR, Jun. 2008

[4] C. Prati, A. Monti Guarnieri, and F. Rocca, "SPOT mode SAR focusing with the $\omega-k$ technique," in Proc. IEEE IGARSS, Espoo, Finland, Jul. 3-6, 1991, pp. 631-634.

[5] A. Monti Guarnieri and C. Prati, "ScanSAR focusing and interferometry," IEEE Trans. Geosci. Remote Sens., vol. 34, no. 4, pp. 1029-1038, Jul. 1996.

[6] A. Moreira, J. Mittermayer, and R. Scheiber, "Extended chirp scaling algorithm for air- and spaceborne SAR data processing in stripmap and ScanSAR imaging modes," IEEE Trans. Geosci. Remote Sens., vol. 34, no. 5, pp. 1123-1136, Sep. 1996.

[7] J. Mittermayer, A. Moreira, and O. Loffeld, "Spotlight SAR data processing using the frequency scaling algorithm," IEEE Trans. Geosci. Remote Sens., vol. 37, no. 5, pp. 2198-2214, Sep. 1999.

[8] R. Lanari, S. Hensley, and P. Rosen, "Chirp Z-transform based SPECAN approach for phase-preserving ScanSAR image generation," Proc. Inst. Elect. Eng.-Radar Sonar Navig., vol. 145, no. 5, pp. 254-261, Oct. 1998.

[9] R. Lanari, M. Tesauro, E. Sansosti, and G. Fornaro, "Spotlight SAR data focusing based on a two-step processing approach," IEEE Trans. Geosci. Remote Sens., vol. 39, no. 9, pp. 1993-2004, Sep. 2001.

[10] A. Monti Guarnieri and P. Guccione, "Optimal 'focusing' for low resolution ScanSAR," IEEE Trans. Geosci. Remote Sens., vol. 39, no. 3, pp. 479-491, Mar. 2001.

[11] D. P. Belcher and C. J. Baker, "High resolution processing of hybrid strip-map/spotlight mode SAR," Proc. Inst. Elect. Eng.-Radar Sonar Navig., vol. 143, no. 6, pp. 366-374, Dec. 1996.

[12] J. Mittermayer, R. Lord, and E. Boerner, "Sliding spotlight SAR processing for TerraSAR-X using a new formulation of the extended chirp scaling algorithm," in Proc. IEEE IGARSS, Toulouse, France, Jul. 21-25, 2003, vol. 3, pp. 1462-1464.

[13] Y. L. Neo, F. Wong, and I. G. Cumming, "A two-dimensional spectrum for bistatic SAR processing using series reversion," IEEE Geosci. Remote Sens. Lett., vol. 4, no. 1, pp. 93-96, Jan. 2007.

[14] D. D'Aria and A. Monti Guarnieri, "High-resolution spaceborne SAR focusing by SVD-Stolt," IEEE Geosci. Remote Sens. Lett., vol. 4, no. 4, pp. 639-643, Oct. 2007.

[15] R. Bamler, F. Meyer, and W. Liebhart, "Processing of bistatic SAR data from quasi-stationary configurations," IEEE Trans. Geosci. Remote Sens., vol. 45 , no. 11 , pp. 3350-3358, Nov. 2007.
[16] A. Moreira, R. Scheiber, and J. Mittermayer, "Azimuth and range scaling for SAR and ScanSAR processing," in Proc. IEEE IGARSS, Lincoln, NE, May 27-31, 1996, pp. 1214-1216.

[17] K. Tomiyasu, "Conceptual performance of a satellite borne, wide swath synthetic aperture radar," IEEE Trans. Geosci. Remote Sens., vol. GRS-19, no. 2, pp. 108-116, Jan. 1981.

[18] C. Y. Chang, M. Y. Jin, Y. L. Lou, and B. Holt, "First SIR-C ScanSAR results," IEEE Trans. Geosci. Remote Sens., vol. 34, no. 5, pp. 1278-1281, Sep. 1996.

[19] R. K. Raney, H. Runge, R. Bamler, I. G. Cumming, and F. H. Wong, "Precision SAR processing using chirp scaling," IEEE Trans. Geosci. Remote Sens., vol. 32, no. 4, pp. 786-799, Jul. 1994.

[20] A. Meta, P. Prats, U. Steinbrecher, J. Mittermayer, and R. Scheiber, "First TOPSAR image and interferometry results with TerraSAR-X," in Proc. Fringe Workshop, Frascati, Italy, Nov. 2007.

[21] W. G. Carrara, R. S. Goodman, and R. M. Majewski, Spotlight Synthetic Aperture Radar: Signal Processing Algorithms (IPF). Norwood, MA: Artech House, 1995.

[22] M. Born and E. Wolf, Principles of Optics, 5th ed. Oxford, U.K.: Pergamon, 1975.

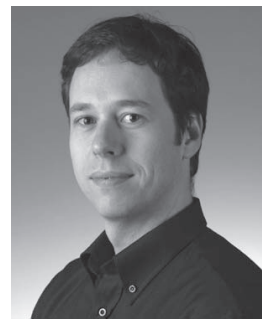

Pau Prats (S'03-M'06) was born in Madrid, Spain, in 1977. He received the M.S. degree in telecommunication engineering and the Ph.D. degree from the Universitat Politècnica de Catalunya (UPC), Barcelona, Spain, in 2001 and 2006, respectively.

In 2001, he was with the Institute of Geomatics, Spain, as a Research Assistant, where he designed a subaperture synthetic aperture radar (SAR) processor. In 2002, he was with the Department of Signal Theory and Communications, UPC, where he worked in the field of airborne repeat-pass interferometry and airborne differential SAR interferometry. From December 2002 to August 2006, he was an Assistant Professor with the Department of Telecommunications and Systems Engineering, Universitat Autònoma de Barcelona, Barcelona. In 2006, he joined the Microwaves and Radar Institute, German Aerospace Center (DLR), Wessling, Germany, where, since August 2009, he is head of the Multimodal Algorithms Group. His research interests include air- and spaceborne SAR processing, SAR interferometry, differential SAR interferometry and motion compensation for airborne systems.

Dr. Prats was the recipient of the First Prize of the Student Paper Competition of the IEEE International Geoscience and Remote Sensing Symposium 2005 Conference held in Seoul, Korea.

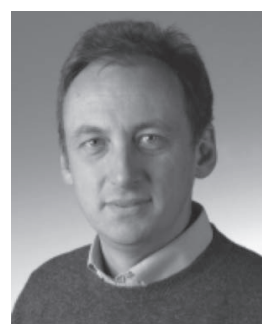

Rolf Scheiber received the M.S. degree in electrical engineering from the Technical University of Munich, Munich, Germany, in 1994 and the Ph.D. degree in electrical engineering from the University of Karlsruhe, Karlsruhe, Germany, in 2003, with a thesis on airborne synthetic aperture radar (SAR) interferometry.

Since 1994, he has been with the Microwaves and Radar Institute, German Aerospace Center (DLR), Wessling, where he developed the operational highprecision interferometric SAR processor for its E-SAR airborne sensor. Since 2001, he has been heading the SAR Signal Processing Group, SAR Technology Department, where he is currently responsible for the E-SAR campaign data processing as well as the development of the processing software for the new airborne sensor F-SAR. His current research interests include algorithm development for high-resolution air- and spaceborne SAR focusing, SAR interferometry, differential SAR interferometry, and SAR tomography, as well as radio sounding algorithms and applications.

Dr. Scheiber was awarded as a coauthor with the 1996 GRSS Transactions Prize Paper Award for the contribution "Extended Chirp Scaling Algorithm for Air- and Spaceborne SAR Data Processing in Stripmap and ScanSAR Imaging Mode" in 1997. 


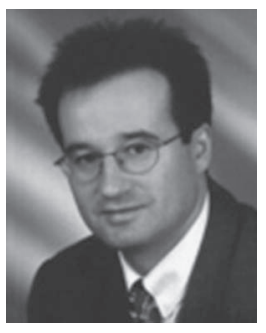

Josef Mittermayer (M'07) was born in Wartenberg, Oberbayern, Germany, in 1967. He received the M.S. degree in electrical engineering from the Technical University of Munich, Munich, Germany, in 1995, with a thesis on ScanSAR processing, the Ph.D. degree in spotlight synthetic aperture radar (SAR) processing from the University of Siegen, Siegen, Germany, in 2000, and the M.S. degree in space system engineering from the Delft University of Technology, Delft, The Netherlands, in 2004.

From 1994 to 2001, he was with the Signal Processing Group, Microwaves and Radar Institute, German Aerospace Center (DLR), Wessling, where, since 2002, he has been working in the TerraSAR Project and, since July 2008, has been leading the SAR System Engineering Group. From January 2004 until the end of the commissioning phase in 2008, he was the Group Leader and the Project Manager of System Engineering and Calibration, one of the three subprojects that form the TerraSAR-X Ground Segment. In addition, he was technically responsible for the TerraSAR-X commissioning phase.

Dr. Mittermayer and his colleagues were the recipient of the Geoscience and Remote Sensing Society Transactions Prize Paper Award for a paper on airand spaceborne stripmap and ScanSAR processing in 1996. In 2001, he was the recipient of the DLR Science Award for his work on spotlight SAR processing.

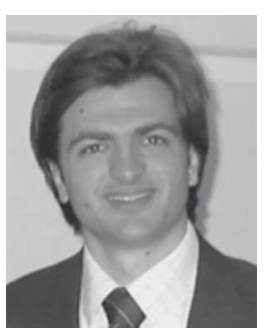

Adriano Meta (S'01-M'05) was born in Pontecorvo, Italy, in 1978. He received the Laurea degree in telecommunication engineering from the University of Rome "La Sapienza," Rome, Italy, in 2002, with the maximum grade and within the best $0.5 \%$ of the students, and the Ph.D. degree (cum laude) from the Delft University of Technology, Delft, The Netherlands, in 2006.

In 2000, he spent an academic year with Ghent University, Ghent, Belgium. From 2002 to 2006, he was with the International Research Centre for Telecommunications and Radar, Delft University of Technology. He has attended the post-lauream Aerospace School, University of Rome "La Sapienza." From 2006 to 2008, he was with the Microwaves and Radar Institute, German Aerospace Center (DLR), Munich, Germany, where he was involved in the commissioning phase of TerraSAR-X and in the development of advanced processing techniques for digital beamforming and multichannel radar applications. He was the Deputy Manager of the demonstration project of the TOPSAR acquisition mode with TerraSAR-X. He is currently the Director with MetaSensing, Noordwijk, The Netherlands, a Dutch company that he founded in 2008 to develop and commercialize compact high-resolution lowcost airborne synthetic aperture radar (SAR) sensors and services. MetaSensing is supported by the European Space Agency through the Business Incubation Program. He is the author of several papers on frequency-modulated continuous-wave (FMCW) SAR signal processing, advanced SAR techniques, and hardware design. He is the holder of an international patent on FMCW SAR frequency nonlinearity correction.

Dr. Meta was the recipient of the Student Paper prizes at the 2006 Waveform Diversity and Design Conference, the 2006 European Conference on Synthetic Aperture Radar, the 2006 IEEE Geoscience and Remote Sensing Symposium, and the 2006 European Radar Conference.

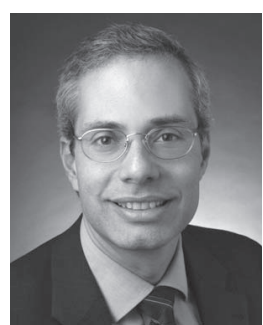

Alberto Moreira (M'92-SM'96-F'04) was born in São José dos Campos, Brazil, in 1962. He received the B.S.E.E. and M.S.E.E. degrees from the Aeronautical Technological Institute (ITA), São José dos Campos, Brazil, in 1984 and 1986, respectively, and the Eng.Dr. degree (with honors) from the Technical University of Munich, Munich, Germany, 1993.

From 1996 to 2001, he was the Chief Scientist and an Engineer with the SAR Technology Department, Microwaves and Radar Institute, German Aerospace Center (DLR), Wessling, where, since 2001, he has been the Director of the Microwaves and Radar Institute. The institute contributes to several scientific programs and space projects for actual and future air- and spaceborne synthetic aperture radar (SAR) missions like TerraSAR-X and Sentinel-1. Under his leadership, the DLR airborne SAR system, E-SAR, has been upgraded to operate in innovative imaging modes like polarimetric SAR interferometry and SAR tomography. In 2003, he became a Full Professor with the University of Karlsruhe, Karlsruhe, Germany, in the field of microwave remote sensing. In 2006, the mission proposal TanDEM-X led by his institute was approved for the realization phase, where he is the Principal Investigator for this mission. He has more than 250 publications in international conferences and journals and is the holder of 15 patents in radar and antenna field. His professional interests and research areas encompass radar end-to-end system design and analysis, innovative microwave techniques and system concepts, signal processing, and remote sensing applications.

Prof. Moreira is serving as a member of the IEEE Geoscience and Remote Sensing Society (IGRSS) Administrative Committee (1999-2001, 2004-2007, and 2008-2009 as the Executive Vice President), was the Founder and Chair of the German Chapter of the GRSS (2003-2008), was an Associate Editor for the IEEE GEOSCIENCE AND REMOTE SENSING LeTters (2003-2007), and has been serving since 2005 as an Associate and Guest Editor for the IEEE Transactions on Geoscience And Remote SEnsing. From 2003 to 2008, he served as a member of the Board of Directors of the Information Technology Society of the German Association for Electrical, Electronic and Information Technologies (VDE). In 1995, he was the recipient of the DLR Science Award. He has contributed to the successful series of the European SAR conferences (EUSAR) since 1996 as a member of the Technical Program Committee, the Technical Chairman (2000), the Awards Chairman (2002-2004), the General Chairman (2006), and the Cochairman (2008). He has participated in 19 IGARSS conferences. He and his colleagues were the recipient of the GRSS Transactions Prize Paper Awards in 1997, 2001, and 2007. He was also the recipient of the IEEE Nathanson Award for the Young Radar Engineer of the Year (1999) and the IEEE Kiyo Tomiyasu Field Award (2007). 